\title{
(In)Visibilité de l'art sur les réseaux sociaux numériques (RSN) : analyser l'acceptation des RSN par les artistes
}

The (in)visibility of art on social network sites: analysis of SNS acceptance

by artists

Amandine Porcher, Jacqueline Vacherand-Revel, Marc-Éric

Bobillier Chaumon, Manon Moktari et Bruno Cuvillier

\section{OpenEdition}

Journals

Édition électronique

URL : http://journals.openedition.org/activites/2805

DOI : 10.4000/activites.2805

ISSN : 1765-2723

Éditeur

ARPACT - Association Recherches et Pratiques sur les ACTivités

Référence électronique

Amandine Porcher, Jacqueline Vacherand-Revel, Marc-Éric Bobillier Chaumon, Manon Moktari et Bruno Cuvillier, « (In)Visibilité de l'art sur les réseaux sociaux numériques (RSN) : analyser

l'acceptation des RSN par les artistes ", Activités [En ligne], 13-2 | 2016, mis en ligne le 15 octobre 2016, consulté le 19 avril 2019. URL : http://journals.openedition.org/activites/2805 ; DOI : 10.4000/ activites.2805

Ce document a été généré automatiquement le 19 avril 2019

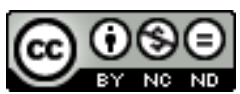

Activités est mis à disposition selon les termes de la licence Creative Commons Attribution - Pas d'Utilisation Commerciale - Pas de Modification 4.0 International. 


\title{
(In)Visibilité de l'art sur les réseaux sociaux numériques (RSN) : analyser l'acceptation des RSN par les artistes
}

\author{
The (in) visibility of art on social network sites: analysis of SNS acceptance \\ by artists
}

Amandine Porcher, Jacqueline Vacherand-Revel, Marc-Éric

Bobillier Chaumon, Manon Moktari et Bruno Cuvillier

\section{NOTE DE L'ÉDITEUR}

Article soumis le 29 janvier 2015, accepté le 29 juin 2016

\section{Introduction 1}

1 Avec le développement des technologies numériques de l'information et de la communication (TICN), les processus à l'œuvre dans le domaine artistique ne semblent plus se dérouler exclusivement via les circuits institutionnalisés traditionnels. Des réseaux plus informels et décentralisés, comme les réseaux sociaux numériques (RSN), rendent possibles de nouvelles formes de production, diffusion, consommation et prescription culturelles. L'usage de ces RSN pourrait alors affecter et transformer les pratiques des artistes.

2 Le métier d'artiste est bien souvent associé au mythe du «don divin » de créativité (Bonnardel, 2009, p. 7) et à des représentations sociales valorisant un style de vie bohème (Bain, 2005) et singulier (Heinich, 1995). Ce travail s'inscrit néanmoins dans une réalité sociale, économique ou encore politique. Un ensemble de déterminants sociaux ${ }^{2}$ participe 
aux carrières de l'artiste et de l'œuvre (soit le «processus qui fait passer l'œuvre par les différentes étapes de son évaluation, attribution, valorisation ", Fourmentraux, 2010, p. 36). Dans ce contexte systémique, il faut pouvoir s'intégrer et développer « un style individuel spécifique et pouvant être commercialisé »" (Bain, ibid., p. 29) si l'on souhaite exercer l'art comme profession. Cependant, vivre de son art est peu courant en raison de diverses difficultés objectives et subjectives (Buscatto, 2006) comme la faible reconnaissance sociale et économique du travail artistique. Cela pousse régulièrement les artistes à trouver des compromis coûteux, comme occuper un emploi "alimentaire " pour survivre, au détriment de leur disponibilité pour l'art.

3 Face à un besoin presque vital de valorisation de l'art, les TICN pourraient représenter diverses opportunités. Les RSN constituent des dispositifs numériques qui permettent la présentation de soi et de son travail artistique en autorisant différentes modalités d'interactions, synchrones ou asynchrones, avec d'autres internautes. Les créateurs peuvent y trouver une vitrine d'échange et d'information se substituant en partie aux médias classiques, développer leur réseau de contacts en lien plus direct avec leur public ou d'autres acteurs culturels (tourneurs, galeristes...), voire développer de nouvelles formes d'art "par, pour et avec » le réseau, dénommé du Netart (Fourmentraux, 2008, p. 58).

4 Ainsi, les RSN peuvent être considérés comme des instruments médiateurs (Rabardel, 1995) de l'activité d'exposition de soi et de ses œuvres. En accédant au statut «d'entité intermédiaire » (Rabardel, ibid.) entre le sujet et l'objet de son action, nous envisageons que ces instruments, à la fois moyen d'action transformatrice ou de connaissance, puissent être porteurs de nouvelles ressources pour une mise en visibilité de l'art et de l'artiste. Cependant, il est aussi possible qu'en situation d'usage réel, les RSN se révèlent contraignants au point d'imposer à l'artiste de modifier ses pratiques, voire d'empêcher son activité d'exposition. Cette dernière pouvant être considérée, à la suite de Clot $(1999,2008)$ comme une épreuve subjective où l'artiste se mesure à soi-même et aux autres. Les RSN ne sont donc pas forcément des technologies acceptables du point de vue de l'artiste. À partir d'une démarche de recherche qualitative, nous identifierons les facteurs favorables ou non à "l'acceptation » des RSN par les artistes engagés dans une activité de «mise en visibilité ». Ces facteurs seront ordonnés au travers des quatre dimensions du modèle de «l'acceptation située» des technologies (Bobillier Chaumon, 2013).

\section{Acceptation des RSN pour ( $\left.s^{\prime}\right)$ exposer}

\subsection{Activité de « mise en visibilité » de l'artiste : enjeux et limites}

5 Pour l'artiste, se « rendre visible » est une activité essentielle, voire vitale. Cette activité est une part inhérente du travail artistique et repose traditionnellement sur le recours à un réseau professionnel et à des lieux physiques dédiés. Par exemple, un artiste peintre aura recours à un galeriste pour exposer ses toiles en galerie ou encore, un chanteur aura recours à un tourneur pour proposer sa performance dans une salle de concert. Dans ces lieux, une scénographie est organisée, bien souvent collectivement, pour mettre en valeur l'œuvre, l'artiste et parfois même le processus de création sous-jacent à la production de l'œuvre (notons que dans certains domaines artistiques, le processus même fait œuvre). 
Néanmoins, cette organisation collective et institutionnalisée de la «mise en visibilité » des artistes bénéficie plus aisément à ceux qui jouissent d'une notoriété suffisante.

6 La «mise en visibilité » recouvre aussi des enjeux économiques, identitaires et de motivation au travail. Dans un contexte socio-économique complexe, exposer crée une opportunité de vendre ou de conclure un contrat. Quelle que soit la forme que prend l'exposition, il s'agit aussi d'une mise en valeur de l'œuvre et du style de l'artiste. L'enjeu identitaire est important pour l'artiste professionnel qui cherche à se distinguer des amateurs, mais aussi des autres artistes par la construction de son identité artistique en autodidacte (Buscatto, 2004), en «stratégie de distinction » (Bourdieu, 1979), en « régime de singularité » (Heinich, 1995). Cette singularisation sera d'ailleurs sujette à l'évaluation et à la reconnaissance des pairs, ce qui pourra soutenir le sentiment d'appartenance à la profession artistique ainsi que la motivation.

7 Malgré de forts enjeux liés à la "visibilité ", l'artiste et son art demeurent en partie « invisibles ». En effet, les artistes sont relativement isolés et difficilement identifiables, notamment, parce qu'ils sont nombreux à exercer d'autres professions (Buscatto, 2006), mais aussi parce que la confusion persiste entre activité artistique de loisir et activité artistique professionnelle. Au moins deux facteurs sont susceptibles de brouiller la frontière entre l'art comme travail et l'art comme loisir. Premièrement, il est difficile de « repérer et rendre visibles les moments, les objets (ex: contrat de travail) et les actes (ex: examens, concours...) professionnels» des artistes (Heinich, 1995, p. 508). Deuxièmement, la profession d'artiste engage dans une "passion culturelle exigeante " (Donnat, 2009, p. 83) qui nécessite un haut degré d'autonomie et d'investissement personnel. Cet excédent de soi dans le travail artistique fragilise la frontière «travail hors travail » (Bain, 2005 ; Buscatto, 2006).

8 L'art en tant que travail est également en partie invisible. Tout d'abord, la création est difficile à définir et appréhender, elle fait l'objet de diverses représentations sociales, de la plus mystique à la plus scientifique. Ensuite, c'est bien souvent l'œuvre qu'on expose, mais en tant que produit fini, elle ne montre pas le processus artistique en amont. Parfois long et coûteux (dans toutes les acceptions du terme), il correspond pourtant à une partie essentielle du travail. Notons que, paradoxalement, «l'invisibilité » peut aussi conditionner la valeur symbolique de l'activité artistique. En quelque sorte, le secret et le mystère de la création sont gages de préciosité.

9 Ainsi, entre la nécessaire " visibilité ", les risques liés à "l'invisibilité », mais aussi l'enjeu de préserver secret certains pans de l'art, l'équilibre paraît difficile à construire. Cet équilibre est d'autant plus instable qu'il est soumis à l'influence de plusieurs facteurs qui échappent pour partie au contrôle de l'artiste. Notamment, « une production créative circule nécessairement dans un système de communication de complexité variable. La proposition créative doit alors être acceptée comme telle par son destinataire (...).» (Rouquette, 2007, p. 44). Les Réseaux Sociaux Numériques (RSN) font partie des lieux et espaces composant ce « système de communication » complexe au sein duquel pourrait se jouer l'équilibre de la « mise en visibilité » de l'art(iste).

\section{2. ( $\left.\mathrm{S}^{\prime}\right)$ Exposer sur les RSN}

10 Les RSN constituent «des services web qui permettent aux individus de construire un profil public ou semi-public au sein d'un système, de gérer une liste des utilisateurs avec lesquels ils partagent un lien, de voir et naviguer sur leurs liens et sur ceux établis par les 
autres au sein du système (...).» (Stenger, \& Coutant, 2010, p. 222). L'usage des RSN peut être motivé par les centres d'intérêt des internautes ou encore avoir pour but le réseautage social pour «créer, développer et entretenir un réseau de contacts dans un cadre professionnel» (ibid., p. 221). Divers RSN existent, qui «répondent tous à un objectif central (...) de la gestion de son image et de ses productions à travers des formes de présence et de « visibilité » (Croissant, \& Touboul, 2011).

11 Pour un artiste, la «mise en visibilité » est une activité à part entière qui réfère à l'ensemble des «mises en scène de soi » au sens de Goffman (1973) et des œuvres, ainsi qu'aux opérations pratiques que ces «mises en scène" nécessitent. Il peut y avoir plusieurs modalités et buts à la " mise en visibilité » sur les RSN. Une "visibilité » sociale participe à se construire socialement "comme artiste en élaborant une identité numérique » et à "construire son public en travaillant son rôle au sein des réseaux interconnectés " (Croissant, \& Touboul, op.cit. p. 6). L'artiste peut signifier aux yeux d'autrui ce qu'il fait, qui il est et élargir l'espace dans lequel manifester sa singularité pour la faire reconnaître (Brodin, \& Magnier, 2012, p. 150-151). Une "visibilité » des créations donne une valeur symbolique et/ou pécuniaire aux œuvres. Une « visibilité » du processus créatif « en train de se faire » est possible quand le RSN est investi comme un espace de création nouveau.

Plus globalement, l'expression et les interactions sur les RSN favorisent la « visibilité » du propos esthétique de l'artiste. En somme, l'usage du RSN peut soutenir la «mise en visibilité » de l'artiste, de ses œuvres, de son travail artistique (en dehors du RSN et éventuellement tel qu'il se pratique sur l'espace numérique). Ces formes de «visibilité numérique " de l'art et de l'artiste servent les enjeux identitaires, de socialisation, économiques et motivationnels présentés précédemment. Cependant, les activités de mises en visibilité numériques ne se transposent pas à celles qui se déroulent hors des médias sociaux. Les lieux, temporalités et acteurs participants de cette visibilité ainsi que les objets visibles diffèrent. Autrement dit, sur les RSN, l'espace de visibilité est immatériel et décloisonné, la durée et les moments de l'accès à l'exposition s'étendent, les interactions avec le public sont plus directes en l'absence de médiation par un tiers professionnel, l'image d'une œuvre n'est pas l'œuvre.

13 Cardon (2008) propose cinq « formats de visibilité » sur les RSN (" paravent, clair-obscur, phare, post-it, lanterna magica ») correspondant aux « différentes formes d'éclairage que les plateformes réservent à l'identité des participants et à leur mise en relation ». Le format " phare » nous semble particulièrement pertinent pour qualifier les pratiques des artistes sur les $\mathrm{RSN}^{4}$ dans la mesure où il correspond au fait de rendre volontairement visibles à un grand nombre d'individus des aspects de leur identité et de leurs productions dans le but de susciter des réactions. Des indicateurs d'audience, de notoriété et un comptage des contacts par les systèmes de RSN objectivent le niveau de «visibilité " alors atteint. Le format «phare » est une forme de « visibilité » instrumentale (Brodin, \& Magnier, 2012) et stratégique, « dont la finalité est de contraindre autrui à l'attention et de susciter son adhésion immédiate » ce qui n'est pas sans risque pour l'artiste.

Cette démarche stratégique implique d'utiliser des techniques de recherche d'attention, de normalisation de soi (ibid., p. 150) ainsi que de mêler « de plus en plus fort les "vrais amis" aux "amis utiles" (...) ». Or, dans cette logique opportuniste et calculatrice, deux limites apparaissent pour l'artiste utilisateur de RSN. Premièrement, il risque de développer un profil stéréotypé influencé par les profils préexistants et par les RSN qui agissent parfois comme de « véritables dispositifs configurants en caractérisant le type de 
liens que peuvent entretenir les individus et en imposant un style d'expression, un marquage temporel... » (Croissant, \& Touboul, 2011, p. 8-9). En outre, un profil stéréotypé s'oppose à la recherche de singularisation et d'expression authentique valorisées au sein de la communauté artistique. Deuxièmement, malgré une démarche intentionnellement stratégique, l'identité numérique créée n'est pas stable et échappe au contrôle total de l'internaute. Les activités sur les RSN « laissent des traces à caractéristiques dont on n'a sans doute pas encore mesuré toute la portée " (Zammar, 2012, p. 274). Ainsi, les comportements des utilisateurs (navigation, commentaires, tags...) produisent des « agrégats " (Cardon, 2008), des traces, dont la "perdurabilité » est certainement une opportunité pour préserver la "qualité des communications asynchrones» (Zammar, 2012, p. 300), mais qui présentent aussi un risque pour l'artiste (par exemple, lorsqu'a posteriori, il ne souhaite plus soutenir une ancienne communication, qu'elle lui semble décalée en regard de sa production actuelle). Ce risque est d'autant plus fort quand l'artiste gère seul sa carrière et sa « visibilité » et/ou qu'il n'a pas une maîtrise experte de l'usage des outils multimédias.

15 En conclusion, l'activité de mise en visibilité réfère à l'ensemble des actions, organisées individuellement et/ou collectivement, au sein des réseaux culturels et artistiques institutionnalisés et/ou non (ex: RSN), qui permettent «la mise en scène » et la présentation des dimensions sociales du métier d'artiste, les dimensions identitaires de l'artiste, les produits de la création ainsi que les processus créatifs sous-jacents. Le but étant de répondre aux enjeux liés à « la visibilité et à l'invisibilité » de l'art et de l'artiste.

\subsection{Processus « d'acceptation » des technologies}

16 En tant qu'artefacts (Norman, 1993), les RSN présentent donc a priori des contraintes et offrent des possibilités dont l'artiste peut se saisir. Il pourra ainsi spécifier ou enrichir les propriétés des RSN jusqu'à ce qu'ils deviennent de réels moyens pour agir: des instruments de "médiation pragmatique ou épistémique » au sens de Rabardel (1995). Cependant, nous avons pu comprendre que les RSN représentent aussi des risques dans le cadre d'une activité de "mise en visibilité ». «L'acceptation» de ces dispositifs par les artistes ne peut donc pas être envisagée comme un acquis, mais comme un construit influencé par des facteurs intrinsèques et extrinsèques au sujet.

Plusieurs modèles de « l'acceptabilité » permettent de repérer des facteurs individuels et sociaux participant à la formation des intentions d'usage d'une technologie, avant même son adoption. "L'acceptabilité » étudiée sous l'angle social et ergonomique vise principalement l'anticipation des comportements d'usage. Les modèles de «l'acceptation » s'inscrivent quant à eux dans le cadre des théories de l'action et dans une approche du « réel » de l'activité (Clot, 1999) lorsque la technologie est effectivement utilisée. L'utilisateur est alors davantage conceptualisé comme un acteur inscrit physiquement, socialement, historiquement et culturellement dans un contexte singulier (Bobillier Chaumon, \& Dubois, 2009, p. 362) : « Appréhender l'utilisateur comme un acteur suppose de le considérer, notamment, en référence à sa culture, son histoire, à la complexité des situations sociales et à la singularité du contexte à l'intérieur duquel il organise son action et insère son activité. » (Bobillier Chaumon, Carvallo, Tarpin-Bernard, \& Vacherand-Revel, 2005, p. 104). Selon cette approche, l'acceptation ou le refus d'une technologie ne sont jamais joués une fois pour toute, une part d'imprévisible demeure. Par exemple, « d'objet initialement rejeté ou délaissé, ce dernier peut, au fil de l'usage et 
des ajustements qu'il subit, devenir attractif et se voir réinvesti d'une nouvelle signification d'usage qui le rend plus acceptable dans la situation. » (Bobillier Chaumon, 2013, p. 51). Ainsi, ce modèle de «l'acceptation» se focalise sur «le déploiement d'un ensemble de conduites réelles d'adoption mises en œuvre par l'individu ou le collectif, dans le cadre des activités effectives au sein de l'organisation" (Bobillier Chaumon \& Dubois, 2009, p. 362). Notons que les concepts « d'acceptabilité » et «d'acceptation » ne s'excluent pas, au contraire, ils se complètent dans l'étude des conceptions, de l'appropriation ou du rejet d'un dispositif technique.

Selon Bobillier Chaumon (2013), l'acceptation se construit dans les moments de l'utilisation effective des TIC, dans le cadre situé, systémique et réel de l'activité (pp. 45-70). "L'acceptation située » vise alors moins à "examiner les conditions d'acceptation de l'objet technologique lui-même que de s'intéresser aux conditions d'acceptation des nouvelles pratiques (ou de la transformation des anciennes, voire de leur empêchement) qui sont liées ou induites par l'usage des technologies. On regarde ainsi très concrètement ce que la technologie permet/autorise de faire, ou oblige à faire, mais aussi ce qu'elle empêche de faire ou plus comme avant et ce, sur différentes dimensions de l'activité" (Bobillier Chaumon, 2016). Précisément, ce sont quatre dimensions (cf. Tableau 1, ci-dessous) qui font l'objet d'une analyse dans le cadre de «l'acceptation située» (ibid., pp. 156-167, voir aussi Bobillier Chaumon, \& Dubois, 2009, pp. 362-373). Au travers d'une première dimension " personnelle ", on interroge les coûts cognitifs et émotionnels liés à l'utilisation d'un dispositif technologique. Avec la dimension "interpersonnelle », on s'intéresse aux reconfigurations des réseaux et des collectifs de travail, car l'introduction d'une TIC peut «redessiner les circuits d'informations, redéfinir les liens de subordination (...).» (Bobillier Chaumon, \& Dubois, ibid., p. 365). Troisièmement, la dimension « méta-personnelle » engage à comprendre les incidences organisationnelles liées, notamment, à la «modification du système de contrôle et d'autonomie des salariés" (ibid., p. 366). Enfin, la dimension «transpersonnelle» considère les constructions professionnelles et identitaires: " l'acceptation ou le refus est fondamentalement lié à une affirmation d'une identité qui s'inscrit toujours dans une culture professionnelle donnée » (ibid., p. 369). 
Tableau 1 : Principales dimensions de « l'acceptation située » (à partir de Bobillier Chaumon, 2013, p. 156).

Table 1 : Main dimensions of situated technology acceptance (Bobillier Chaumon, 2013, p. 156)

\begin{tabular}{|c|c|c|}
\hline $\begin{array}{l}\text { Dimensions de } \\
\text { l'acceptation }\end{array}$ & Définitions & Facteurs pouvant être repérés \\
\hline \multirow[t]{2}{*}{ Personnelle } & \multirow[t]{2}{*}{$\begin{array}{l}\text { Coûts cognitifs et émotionnels liés à } \\
\text { I'utilisation des technologies }\end{array}$} & $\begin{array}{l}\text { Cognitifs : densification de l'activité, surcharge } \\
\text { quantitative, intensification, sous-charge } \\
\text { qualitative et/ou quantitative, (im)possibilité de } \\
\text { transferts d'apprentissage. }\end{array}$ \\
\hline & & $\begin{array}{l}\text { Emotionnels : inconfort à mal-être, émotion } \\
\text { prescrite/empêchée. }\end{array}$ \\
\hline Interpersonnelle & $\begin{array}{l}\text { Reconfigurations en œuvre sur les } \\
\text { collectifs et réseaux de travail. }\end{array}$ & $\begin{array}{l}\text { Redéfinition du collectif de travail } \\
\text { (enrichissement versus affaiblissement), } \\
\text { transformations du travail collectif. }\end{array}$ \\
\hline Méta-personnelle & $\begin{array}{l}\text { Incidences socio-organisationnelles } \\
\text { des technologies sur l'usager et son } \\
\text { activité }\end{array}$ & $\begin{array}{l}\text { Régulation et prescription de l'activité, } \\
\text { supervision ou contrôle sur l'individu, autonomie } \\
\text { imposée, gains/pertes de pouvoir et de marges } \\
\text { de manœuvres. }\end{array}$ \\
\hline Transpersonnelle & $\begin{array}{l}\text { Répercussions sur la construction et } \\
\text { la reconnaissance identitaires de } \\
\text { l'individu }\end{array}$ & $\begin{array}{l}\text { Sens et reconnaissance au travail, } \\
\text { développement/empêchement de l'activité, } \\
\text { requalification / déqualification / } \\
\text { disqualification. }\end{array}$ \\
\hline
\end{tabular}

\section{Problématique et méthodologie}

Dans certaines conditions, les RSN pourraient soutenir ou entraver l'activité de mise en visibilité des œuvres, des propos esthétiques, des processus créatifs ou encore des artistes eux-mêmes. Cependant, les RSN ne sont pas d'emblée des instruments « acceptables » et " acceptés " par les artistes. Afin d'identifier et comprendre les facteurs soutenant ou entravant leur "acceptation", quinze artistes ont été sollicités pour une ou deux entrevues. Douze entretiens semi-directifs nous ont permis d'appréhender leurs métier et activité artistique ${ }^{5}$ ainsi que d'accéder à une compréhension de leurs représentations et usages des TICN, particulièrement des RSN. Afin d'approfondir certaines dimensions émergeant de l'analyse thématique, quatre entretiens à visée d'explicitation ont ensuite été menés au sujet d'une expérience vécue spécifique.

\subsection{Population}

Cette enquête s'appuie sur la participation de quinze artistes traditionnels et numériques, comme le montre le tableau 2, ci-dessous. Cet échantillon de convenance compte 9 hommes et 6 femmes. Neuf participants exercent l'art en tant qu'activité professionnelle à part entière, sans nécessairement parvenir à en tirer une rémunération. Ils sont identifiés par l'abréviation pro. Les six autres participants réalisent des activités quasiprofessionnelles (exposition, promotion, vente...), mais exercent un métier différent. Ils sont qualifiés de pro-am., pour " professionnels-amateurs ». Les pro-am occupent un statut de « quasi-créateur» (Bacache, Bourreau, Gensollen, \& Moreau, 2009, p. 13) et déploient leur activité dans le cadre des «standards professionnels » (Flichy, 2010, p. 8). Parmi ces derniers, la majorité aimerait ou rêve d'exercer l'art au quotidien. En attendant, ils sont étudiants (Marie, Télia), kinésithérapeute (Filippo), vendeur (Sonia), documentaliste (Pedro) et auto-entrepreneur (Liam). L'échantillon pour les entretiens à visée d'explicitation compte Serge Emmanuel (sculpteur), Noël (comédien - metteur en scène), 
Christelle (artiste peintre) et Liam (chanteur - compositeur, pro-am). Nous avions déjà interviewé Christelle dans la phase d'entretiens semi-directifs, elle s'était inscrite sur facebook la veille de l'entretien. Il nous intéressait de l'entendre à nouveau après quelques semaines d'utilisation et d'interroger son activité de mise en visibilité médiatisée par le RSN. Serge Emmanuel, nous avait également déjà reçus pour une séance d'expérimentation de la tâche de création dans le cadre d'une analyse de l'activité de création.

La moyenne d'âge de notre population est d'environ 37 ans, et l'ancienneté moyenne dans la pratique de l'art est d'à peine plus de 15 ans. Ce dernier chiffre correspond à des valeurs auto-déclarées par les artistes qui expriment régulièrement un engagement précoce dans l'art. Parmi les exemples les plus forts, Pedro déclare une ancienneté équivalente à son âge et Michel nous fait part d'un récit d'enfance «l'institutrice a accroché un tableau, une reproduction de Van Gogh au mur et j'ai été, j'ai été ému, bouleversé (...) depuis je peins, je n'ai jamais arrêté ». Cet ancrage de l'art dans l'histoire du sujet est cohérent avec d'autres recherches faisant état d'un «naturalisme de l'acte créatif» (Le Coq, 2004) comme une « nécessité intérieure profonde » (Quitaud, 1993) ou encore de l'art comme un « don » (Bonnardel, 2009).

Tableau 2: Synthèse des principales caractéristiques de la population. Table 2 : Main characteristics of population

\begin{tabular}{|c|c|c|c|c|c|}
\hline Id. & Nom (sexe) & Artiste & Statut & Age & Ancienneté \\
\hline A1 & Michel (H) & Artiste peintre & Pro. & 48 & 27 \\
\hline A2 & Marie (F) & Plasticienne & Pro-am. & 25 & 15 \\
\hline A3 & Télia (F) & Chanteuse jazz & Pro-am. & 20 & 10 \\
\hline A4 & Filippo $(\mathrm{H})$ & Artiste peintre & Pro-am. & 47 & 4 \\
\hline A5 & Ben $(\mathrm{H})$ & Artiste numérique (image) & Pro. & 37 & 17 \\
\hline A6 & Christelle (F) & Artiste peintre & Pro. & 53 & 13 \\
\hline A7 & Florie (F) & Sculptrice & Pro. & 35 & 15 \\
\hline A8 & Sonia (F) & Artiste peintre & Pro-am. & 27 & 3 \\
\hline A9 & Florent $(\mathrm{H})$ & Compositeur acousmatique & Pro. & 43 & 22 \\
\hline A10 & Steven $(\mathrm{H})$ & Artiste numérique (musique) & Pro. & 33 & 8 \\
\hline A11 & Pedro $(\mathrm{H})$ & Dessinateur - illustrateur & Pro-am. & 31 & 31 \\
\hline A12 & Clarisse (F) & Chanteuse lyrique & Pro. & 30 & 12 \\
\hline A13 & Serge Emmanuel (H) & Sculpteur & Pro. & 65 & 31 \\
\hline A14 & Noël $(\mathrm{H})$ & Comédien, metteur en scène & Pro. & 30 & 10 \\
\hline A15 & Liam $(\mathrm{H})$ & Chanteur-compositeur & Pro.am & 29 & 11 \\
\hline
\end{tabular}

\subsection{Entretiens semi-directifs et à visée d'explicitation}

En cohérence avec notre problématique de « l'acceptation » des RSN dans cette activité de " visibilité ", nous avons élaboré un guide d'entretien articulé en trois axes thématiques (1. art et processus créatif, 2. représentations et usages des technologies, 3. usage d'internet et des réseaux sociaux numériques) et une conclusion avec des questions d'ouverture (ex: «De votre point de vue, un artiste peut-il se passer d'internet? des RSN?»). Les entretiens ont duré en moyenne une heure et dix minutes. Le principe de la discussion ouverte à partir de ces thématiques était fondamental et a été énoncé systématiquement en consigne inaugurale. Cette posture d'ouverture a été particulièrement importante dans le cadre de cette recherche auprès d'artistes. En effet, 
leur métier et leurs activités sont relativement difficiles à appréhender, notamment, parce qu'ils sont en partie invisibles et singuliers comme nous l'avons vu, mais aussi parce que nous ne disposons que de peu (voire pas) de référentiels de travail à partir desquels nous aurions pu baser notre compréhension. Aussi, les méthodes de recueil de données mises en œuvre dans cette recherche ne sont pas nouvelles, leur originalité réside dans leur application au domaine artistique.

Les entretiens à visée d'explicitation s'inscrivent en complémentarité des entretiens semi-directifs. Dans une logique de triangulation, ils ont permis de confronter les premières analyses réalisées à une nouvelle source de données. Notre méthode a été nourrie des principes de la technique d'entretien d'explicitation (Vermersch, 1994) sans pour autant l'avoir appliquée exhaustivement. Ainsi, les entretiens visaient bien l'explicitation des actions, pensées et activités mentales, perceptions sensorielles et émotions de l'artiste à un moment donné d'une activité spécifique de mise en visibilité. Nous ne cherchions pas principalement la verbalisation des avis et opinions, mais des verbalisations décrivant l'action effective et son vécu (ibid., pp. 33-41). Pour cela, il était important que les personnes se souviennent d'un instant précis. À cette fin, un support mnésique était proposé aux artistes alors que ce type d'aide à la remémoration est proscrit dans la technique développée par Vermersch. Dans les faits, seul l'entretien avec Liam s'est tenu avec un ordinateur et internet afin d'accéder au contenu des RSN utilisés. Les trois autres entretiens se sont déroulés sans support mnésique. Cela a engendré plus d'effort d'explicitation pour nos interlocuteurs avec qui nous avons échangé 62 minutes en moyenne. Les questionnements se sont déroulés en trois temps: amorce de la discussion à propos de leur activité artistique, questions générales sur l'utilisation et le rapport aux RSN, explicitation d'une activité de mise en visibilité précise rappelée librement ou par le biais du support mnésique.

\subsection{Analyse thématique des données}

24 Sur la base des verbatim des entretiens semi-directifs et à visée d'explicitation intégralement retranscrits, nous avons procédé à une analyse thématique. Les résultats ont été intégrés en une structure d'analyse globale (dite « arbre thématique ») relative à l'acceptation des RSN pour (se) mettre en visibilité lorsqu'on est un artiste. L'analyse thématique «se réclame d'une approche subjectiviste et vise à reformuler, à interpréter et à théoriser des phénomènes » (Therriault, \& Harvey, 2011, p. 81). Comme l'illustre la figure 1, nous avons procédé en quatre étapes : (E1) repérer les thèmes représentatifs du contenu analysé, (E2) les regrouper en ensembles thématiques saillants selon leurs similarités (et les distinguer selon leurs différences), (E3) confronter ces ensembles au modèle de référence afin d'identifier les points de correspondance et les résultats émergents hors du modèle, et enfin, (E4) organiser les ensembles thématiques en quatre axes dans un "arbre thématique ", et spécifier les résultats de chacun de ces axes afin de procéder à leur examen discursif (Paillé, \& Mucchielli, 2012, p. 232). Cette démarche a été réalisée entièrement de façon continue et manuscrite. La continuité de l'analyse renvoie à la simultanéité du travail de thématisation et d'intégration des résultats. 
Figure 1 : Méthodologie - Schéma de synthèse des étapes (E) et des axes de l'analyse.

Figure 1 : Methodology - Overview diagram of the stages $(E)$ and outcomes of the data analysis

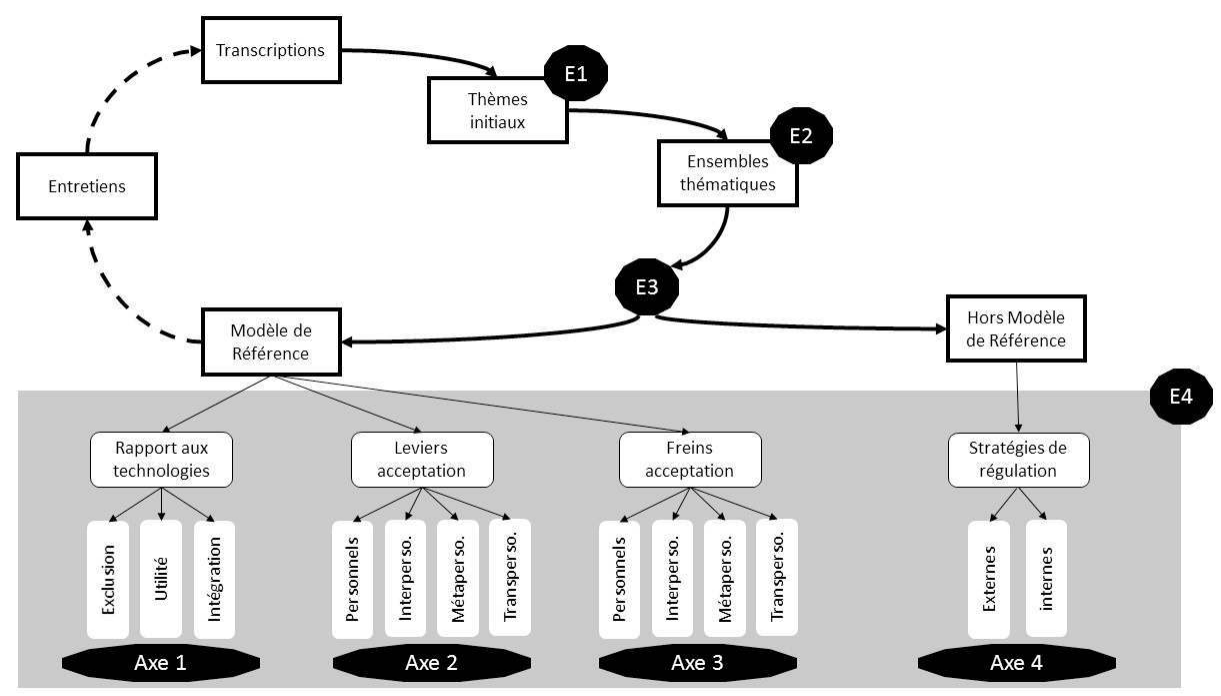

Le premier travail de repérage des thèmes initiaux (E1) a débuté par une lecture flottante des entretiens avec annotations libres. Ensuite, les verbatim ont été découpés en unités de significations. Ces unités se rapportent à la dimension référentielle du discours (Fallery, \& Rodhain, 2007). Elles correspondent à des extraits plus ou moins longs des verbatim. On les repère à chaque transition de thème dans le discours du locuteur. Le thème « renvoie à ce qui est abordé dans l'extrait du corpus correspondant, tout en fournissant des indications sur la teneur des propos. [...] Le thème permet à la fois d'étiqueter et de dénoter un extrait. » (Paillé, \& Mucchielli, 2012, p. 19).

Cette «thématisation » a été guidée par deux interrogations systématiques pour chaque unité de signification: «De quoi est-il question au juste dans l'extrait analysé? Quel thème précis est soulevé ?» (ibid., p. 19). Par exemple, Télia (chanteuse de jazz) dit que sur les RSN « la diffusion est quand même d'une rapidité! On poste une vidéo elle est là, bam, chez tout le monde. On peut cibler énormément de personnes et puis facebook, les gens y vont souvent. Et du monde entier. Oui, un correspondant anglais qui vit en France, bam, des amis anglais voient ça. » Dans cet extrait on comprend qu'un des facteurs favorisant l'usage des RSN pour Télia repose sur la "viralité et rapidité de diffusion» de contenus. Ce thème initial indique que, lors de l'entretien, il a été question de « l'activité de diffusion d'informations sur les RSN ». Elle est ensuite qualifiée de «rapide " grâce au principe de "viralité numérique ». Le thème est donc un outil analytique dirigé vers la compréhension du processus d'acceptation des RSN par les artistes. Par cette démarche, nous cherchons à accéder au sens profond de l'expérience des sujets en lien avec la problématique de recherche.

La seconde étape du travail de "thématisation" a consisté à regrouper les ensembles thématiques saillants (E2). À ce stade, l'analyse continue a transformé les thèmes initiaux et leur hiérarchisation, de nouveaux thèmes sont nés de la fusion ou de la division de thèmes précédents. Le thème illustratif « viralité et rapidité de diffusion » n'apparaît plus en tant que tel. Il a fait l'objet d'une subdivision puis a été rattaché, notamment, à un ensemble thématique alors intitulé "économie cognitive relative à la diffusion de contenus », et à un second ensemble relatif à la « facilitation du réseautage ». 

des RSN par les artistes se structure à partir de quatre axes principaux relevés dans l'analyse. Ainsi, l'acceptation des RSN s'ancre dans un rapport aux technologies qui varie selon les artistes (axe 1), «l'acceptation» est influencée par des facteurs favorables (axe 2), mais aussi par des freins (axe 3) «personnels, interpersonnels, métapersonnels et transpersonnels ». Enfin, le processus «d'acceptation » sous-tend différentes stratégies de régulation des contraintes vécues dans l'usage du réseau social (axe 4).

\subsection{AXE 1 : Rapports aux technologies de l'échantillon d'artistes}

31 Le tableau 3, ci-dessous, indique que les artistes rencontrés utilisent différents types de réseaux numériques. Certains de ces réseaux sont généraux (c'est le cas du RSN Facebook, par exemple) et d'autres sont dédiés à l'art. Si la plupart des réseaux sont utilisés de façon individuelle pour «mettre en visibilité » sa propre activité, sept artistes partagent aussi un réseau avec un collectif d'artistes. La plupart des artistes ont un usage quotidien des RSN, pour autant, ils ne déclarent pas tous se sentir familiers avec les TICN. 
Tableau 3 : pratique des réseaux numériques et sentiment de familiarité. Table 3 : Digital practices and sense of familiarity

\begin{tabular}{|c|c|c|c|c|c|c|c|c|}
\hline \multirow{2}{*}{ Id. } & \multirow{2}{*}{ Art. } & \multicolumn{2}{|c|}{ RSN } & \multicolumn{2}{|c|}{ Site / Blog (pro.) } & \multirow{2}{*}{$\begin{array}{c}\text { Messagerie/ } \\
\text { Courriel-(pro.) }\end{array}$} & \multirow{2}{*}{ Usage } & \multirow{2}{*}{$\begin{array}{c}\begin{array}{c}\text { Sentiment } \\
\text { de }\end{array} \\
\text { Familiarité }\end{array}$} \\
\hline & & Général & Dédié Art & Individuel & Collectif Art & & & \\
\hline A1 & Artiste peintre & & & $\mathrm{x}$ & & & FAIBLE & Non \\
\hline A2 & Plasticienne & $\mathrm{x}$ & $\mathrm{x}$ & $\mathrm{x}$ & $\mathrm{x}$ & $\mathrm{x}$ & QUOTIDIEN & Oui \\
\hline A3 & Chanteuse jazz & $\mathrm{x}$ & $\mathrm{x}$ & $\mathrm{x}$ & $\mathrm{x}$ & $\mathrm{x}$ & MODÉRÉ & Oui \\
\hline A4 & Artiste peintre & & & $\mathrm{x}$ & & $\mathrm{x}$ & MODÉRÉ & Non \\
\hline A5 & $\begin{array}{l}\text { Artiste numérique } \\
\text { (image) }\end{array}$ & $\mathrm{x}$ & $\mathrm{x}$ & $\mathrm{x}$ & $\mathrm{x}$ & $\mathrm{x}$ & QUOTIDIEN & Oui \\
\hline A6 & Artiste peintre & $\mathrm{x}$ & & $\mathrm{x}$ & & $\mathrm{x}$ & QUOTIDIEN & Non \\
\hline A7 & Sculptrice & $\mathrm{x}$ & & $\mathrm{x}$ & & $\mathrm{x}$ & QUOTIDIEN & Non \\
\hline A8 & Artiste peintre & $\mathrm{x}$ & & & & $\mathrm{x}$ & QUOTIDIEN & Non \\
\hline A9 & $\begin{array}{l}\text { Compositeur } \\
\text { acousmatique }\end{array}$ & $\mathrm{x}$ & & $\mathrm{x}$ & $\mathrm{x}$ & $\mathrm{x}$ & QUOTIDIEN & Oui \\
\hline A10 & $\begin{array}{l}\text { Artiste numérique } \\
\text { (musique) }\end{array}$ & $\mathrm{x}$ & $\mathrm{x}$ & $\mathrm{x}$ & $\mathrm{x}$ & $\mathrm{x}$ & QUOTIDIEN & Oui \\
\hline A11 & $\begin{array}{c}\text { Dessinateur - } \\
\text { illustrateur }\end{array}$ & $\mathrm{x}$ & $\mathrm{x}$ & $\mathrm{x}$ & $\mathrm{x}$ & $\mathrm{x}$ & QUOTIDIEN & Oui \\
\hline A12 & Chanteuse lyrique & $\mathrm{x}$ & & & & $\mathrm{x}$ & MODÉRÉ & Oui \\
\hline A13 & Sculpteur & $\mathrm{x}$ & & $\mathrm{x}$ & & $\mathrm{x}$ & QUOTIDIEN & Non \\
\hline A14 & $\begin{array}{l}\text { Comédien, metteur } \\
\text { en scène }\end{array}$ & & $\mathrm{x}$ & & $\mathrm{x}$ & $\mathrm{x}$ & FAIBLE & Non \\
\hline A15 & $\begin{array}{c}\text { Chanteur- } \\
\text { compositeur }\end{array}$ & $\mathrm{x}$ & $\mathrm{x}$ & & & $\mathrm{x}$ & QUOTIDIEN & Oui \\
\hline
\end{tabular}

L'analyse thématique révèle l'importance du rapport général que les artistes développent à l'égard des technologies dans le processus d'acceptation des RSN. Ce processus s'ancre donc dans une trajectoire globale et historique d'usage des technologies. L'analyse fait émerger trois types de rapport aux technologies. Une première partie des artistes a un rapport ambigu aux technologies numériques qu'ils utilisent tout en les rejetant dans leur discours (artistes A1, 4, 12, 14). Notons que pour ces derniers l'usage des RSN est plutôt rare. Dans le tableau 3, ci-dessus, nous avons qualifié leur usage de faible ou modéré. Un à trois maximum types de TICN différentes sont utilisées et seul un artiste se dit familier de leur usage. Les attitudes négatives développées envers ces systèmes sont nourries de leur caractère désincarné, du sentiment de contradiction avec leurs valeurs professionnelles et personnelles, voire de l'absence de plus-value créative et esthétique pour l'art. Les technologies sont éventuellement envisagées positivement, mais c'est à condition que l'artiste n'interagisse pas directement avec elles (utilisation par d'autres artistes, au nom d'un collectif et pas au nom propre de l'artiste, ou encore dans une gestion déléguée de l'artiste à un tiers proche ou professionnel).

Un second groupe d'artistes utilise des technologies pour leurs apports potentiels ou réels (A3, 6, 7, 8,11, 13). Dans le tableau $\mathrm{n}^{\circ} 3$, ci-dessus, nous constatons que, pour ce groupe, un minimum de deux et jusqu'à cinq types de technologies différentes sont utilisées. Un seul artiste dit en avoir un usage modéré, les autres en ont un usage quotidien. Pour autant, ils n'ont pas forcément développé un sentiment de familiarité à leur égard. Globalement, l'attitude envers les technologies est positive, elles sont associées au développement du domaine artistique, de la pratique artistique individuelle et de la valeur (au moins symbolique et esthétique) de certaines œuvres. La technologie peut avoir une place dans leur processus créatif en tant qu'instrument, mais ce n'est pas une nécessité. À ce sujet, nous avons constaté que l'usage des technologies se substitue parfois à celui d'instruments plus traditionnels. Cela permet notamment de faire face à des contraintes irréductibles comme l'impermanence des objets de la création (à entendre au sens large). Un dessin à l'encre sur le papier peut se dégrader dans le temps ou encore le modèle vivant ne peut pas rester physiquement présent à chaque instant où l'on sculpte la terre ; 
en somme, l'encre et le modèle sont de ces objets impermanents de la création. Face à cela, Pedro (illustrateur) réalise parfois la tâche d'encrage sur tablette numérique après avoir scanné son esquisse faite au crayon gris, et Florie (sculptrice) prend occasionnellement des clichés numériques de son modèle. Le recours à la technologie n'est pas le premier réflexe, car ces artistes privilégient le « rapport charnel » (Florie) qui représente une donnée du sensible de la création.

Enfin, pour un dernier groupe (A2, 5, 9, 10, 15), la technologie est un instrument de prédilection intégré à leurs activités et leur démarche artistiques. Comme le tableau n⿳o 3 l'indique, les artistes de ce groupe ont tous une pratique quotidienne avec un usage familier de trois à cinq types de TICN différentes. L'art et la technologie fusionnent en un projet où l'esthétique est à la fois sensible et numérique. La machine tient lieu d'acteur de la création. Ses propriétés et caractéristiques participent à la formulation de la direction artistique et des choix créatifs. Par exemple, les créations de Steven (musicien) dépendent en partie d'un algorithme euclidien. Pour les artistes de ce groupe, les contraintes des technologies sont acceptées, voire valorisées. Elles sont intégrées à leur démarche comme des règles structurant le jeu artistique («les limitations, comme les bords de la feuille sont un cadre, c'est plus facile pour moi d'envisager comme un dessinateur comment disposer le dessin à l'intérieur, les limitations de la machine (...) me permettent de mieux focaliser mon travail. » Steven). Dans les discours, la machine est personnifiée, elle a une vie propre, une personnalité et des limites physiques comme des muscles de danseurs qui parfois peuvent lâcher (selon l'analogie de Ben, artiste image numérique). L'usage des technologies est qualifié de «naturel» et permet une hybridation des matériaux, techniques et acteurs de la création comme lorsque Ben explique "j'ai une plaque de plexiglas sur laquelle je peins en fait, et la vidéo vient se prendre là-dessus et c'est re-filmé pour être projeté sur les danseurs. (...) Là où je mets de la matière, c'est à l'endroit même où le danseur est. »

Les artistes rencontrés n'entretiennent pas un rapport identique aux technologies. Néanmoins, ils ont en commun de disposer d'un espace de (re)présentation numérique (au sein duquel ils sont plus ou moins actifs). Ils sont présents sur les RSN et, à l'unanimité, ils déclarent que cela est indispensable pour un artiste «aujourd'hui». Leur participation à cette recherche a donc été propice à l'identification de facteurs favorables ou non à l'acceptation des RSN pour l'activité qu'ils réalisent et/ou qu'ils cherchent à déployer. Dans les axes 2 et 3 de «l'arbre thématique » mis en évidence par l'analyse, ces facteurs ont été ordonnés selon qu'ils renvoient à la dimension "personnelle, interpersonnelle, métapersonnelle ou transpersonnelle» du modèle "d'acceptation située » des technologies.

\subsection{AXE 2 : leviers « d'acceptation » des RSN}

\subsubsection{Dimension " personnelle » : un soutien cognitif clair, mais une charge émotionnelle variable}

Pour les artistes de notre échantillon, les RSN offrent indéniablement un support cognitif efficient facilitant les activités de recherche et de transmission d'informations. Il devient facile de s'informer, de se former et de s'inspirer grâce à l'accès rapide à une grande quantité de contenus centralisés et organisés. Pour rendre visible un contenu lié à son art, différents RSN se complètent et se relient aisément. Par exemple, il est simple de publier sur facebook un lien vers une vidéo Youtube. La multiplicité des plateformes numériques 
de « réseautage » permet aussi aux artistes d'en faire un usage différencié et distribué. Ils répartissent ainsi leurs contenus et contacts en plusieurs espaces et peuvent dédier chaque RSN à des activités et tâches spécifiques. Enfin, deux derniers éléments allègent la charge cognitive liée à « la mise en visibilité » : la possibilité de publier une information sans adresse directe et de bénéficier ensuite du principe de "viralité » pour que cette même information circule.

Sur le plan émotionnel, il semble que la "présentation de soi " numériquement médiatisée, de ses compétences et du travail "bien fait» crée un sentiment de satisfaction et de fierté. Notons que les artistes rencontrés accentuent leur usage des RSN quand leur carrière est en développement. Il semblerait donc que développement de leur satisfaction professionnelle et de leur satisfaction à l'usage des RSN s'alimentent mutuellement. Par ailleurs, à l'unanimité, les feedbacks proposés par les internautes représentent une source de motivation. Nous constatons également que les artistes construisant activement leur identité et "mise en visibilité » via les RSN, en retirent un sentiment d'auto-efficacité et de contrôle. Ils développent aussi des compétences nouvelles au service de leur "mise en visibilité ». C'est, par exemple, le cas de Steven (musicien) qui a pris conscience de son image «virtuelle » d'artiste via l'usage des RSN, puis a appris à la travailler, alors que, dit-il, c'est une «chose qui n'est pas du tout naturelle pour moi, le travail de l'image, je n'ai pas du tout été formé à ça, j'ai pas de facilité (...) ça m’a poussé à avoir une réflexion là-dessus ». Soulignons que les leviers émotionnels participant de «l'acceptation» des RSN sont des thématiques particulièrement présentes dans le discours des artistes qui ont de l'appétence et sont déjà familiers dans l'usage de diverses technologies numériques.

\subsubsection{Dimension « interpersonnelle » : levier principal dans » l'acceptation » des RSN}

La dimension interpersonnelle est la plus favorable à l'acceptation des RSN. Les avantages de ce "réseautage numérique " se déclinent selon les deux axes de cette dimension proposés par Bobillier Chaumon (2013, cf. tableau oํ1).

Le premier axe concerne la reconfiguration des réseaux de travail. L'acceptation des RSN est favorisée par l'extension des réseaux de contacts et de diffusion. Le "réseautage numérique " se révèle facile et efficace. En effet, l'artiste entre en relation avec des acteurs du secteur, ce qui peut déclencher une vente, développer des partenariats nouveaux, des projets collaboratifs pluridisciplinaires ou aboutir à la signature d'un contrat. Par exemple, Clarisse (chanteuse lyrique) est en contact avec des metteurs en scène, « le fait de voir mes actualités et tout ça, ils voient que oui, je continue à chanter, ça marche bien pour moi, et que oui, ils ont envie de m'embaucher pour les prochains concerts ». Enfin, la possibilité de «voir» et "d'être vu» sans engager pour autant une interaction directe avec des contacts est plébiscitée. La suite des propos de Clarisse illustre ce point :

«c'est vraiment une espèce de maintien du lien social avec des gens que je ne revois pas forcément, mais de qui je prends des nouvelles soit à leur insu, soit réellement $"$.

Le second axe, est relatif à l'évolution du rôle des collectifs de travail. Les interactions avec des pairs artistes apportent soutiens, échanges de pratiques ou conseils. Ce sont ce que Dejours (1995) appelle des « jugements de beauté » qui sont exprimés sur les RSN. Ils participent à la fois à la reconnaissance de l'artiste, à sa motivation et au développement 
d'un sentiment d'appartenance malgré l'absence de rencontres physiques. C'est ce qu'exprime Marie (plasticienne) :

" oui c'est vrai, je me sens aussi appartenir à des groupes sur internet, oui. » ou Steven (musicien) :

«j'ai la sensation, l'illusion d'une forme de localité, en fait, de quartier. (...) ça fait comme un village».

Cependant, les pairs en question sont souvent des internautes inconnus, ce qui rend ces collectifs virtuels de travail particuliers. La rencontre physique reste néanmoins la plus valorisée.

\subsubsection{Moins de leviers « d'acceptation » rattachés aux dimensions « métapersonnelle et transpersonnelle »}

41 Les facteurs "d'acceptation ", identifiés dans cette population, relatifs aux dimensions «métapersonnelle et transpersonnelle» sont moins prégnants que les facteurs "personnels et interpersonnels». L'analyse thématique a fait apparaître que pour la dimension «métapersonnelle» trois incidences organisationnelles des RSN peuvent représenter des facteurs "d'acceptation ». Premièrement, les RSN semblent renforcer l'activité de «mise en visibilité » en s'inscrivant au sein de systèmes d'instruments (avec par exemple, le site web, le mail ou la presse professionnelle). Les RSN se substituent parfois à des activités de promotion en provoquant automatiquement un référencement de la page ou du profil de l'artiste sur des moteurs de recherche généraux et/ou spécifiquement liés à l'art et la culture. Deuxièmement, le fonctionnement des RSN offre des marges de manœuvre quant à l'espace de "visibilité ». Les artistes peuvent contrôler l'étendue de l'espace sur lequel ils souhaitent « être visible » en le limitant à un groupe ou une liste de contact ou, au contraire, en l'ouvrant publiquement sans restriction. Troisièmement, le système contrôle et quantifie l'audience (statistiques, courbes, alertes) ce qui peut utilement être considéré comme un indicateur de notoriété. Le rôle de ces facteurs dans « l'acceptation » des RSN varie selon que les artistes développent un usage novice ou expert des dispositifs.

La dimension «transpersonnelle » réfère au métier, à l'identité professionnelle, au sens au travail et encore à la qualification. De notre analyse, il ressort trois facteurs qui peuvent se révéler intéressants pour favoriser l'acceptation des RSN. Tout d'abord, le métier peut évoluer grâce à de nouveaux matériaux, collectifs ou en raison de la fusion de plusieurs métiers et disciplines sur les RSN. La reconnaissance par les pairs serait favorisée par le partage d'espaces numériques communs et le sentiment d'appartenance déjà évoqué, la reconnaissance par le public serait également améliorée (matérialisée, par exemple, par des systèmes de votes en ligne pour l'artiste inscrit dans un concours). Enfin, nous avons vu que se «mettre en visibilité » fait partie intégrante du "réel de l'activité » des artistes, mais, pour autant, les participants énoncent leur difficulté dans ce domaine (comme Steven (musicien) pour qui le travail de l'image n'est pas «naturel », par exemple). L'acceptation des RSN est alors soutenue lorsque ces dispositifs accompagnent le développement de nouvelles compétences liées à «la mise en visibilité ». 


\subsection{Axe 3 : freins dans le processus "d'acceptation »}

\subsubsection{Les dimensions « personnelle et interpersonnelle » se rejoignent sur le plan de la charge émotionnelle}

Des charges cognitives et émotionnelles liées à l'usage des RSN font peser de fortes contraintes sur le processus «d'acceptation». Sur le plan cognitif, les utilisateurs font état d'une intensification du travail liée, notamment, à des sollicitations accrues de la part d'« amis virtuels» (selon les termes de Michel, artiste-peintre), mais aussi de la part du système de RSN qui exige des mises à jour, rappelle les messages en attente, propose des évolutions... (cf. la dimension «métapersonnelle»). Les artistes doutent de leur capacité à tenir la cadence rapide de publication censée répondre à ces sollicitations. Nous constatons que «(se) mettre en visibilité » relève de compétences et raisonnements spécifiques qui demandent à être développées. Aussi, l'effort d'apprentissage peut s'accompagner d'un faible sentiment d'auto-efficacité, en particulier pour les plus novices (surtout lors de mises en échec face aux exigences techniques du système). La charge cognitive augmente également face aux contraintes ou défaillances du système. Enfin, quand les artistes utilisent plusieurs RSN, ils redoutent ou expérimentent une dispersion de leur image, leur présence devient moins lisible et floue ce qui nuit de facto à leur «visibilité ».

Des tensions émotionnelles freineraient « l'acceptation » des RSN. Des conflits de valeurs personnelles émergent lorsque l'utilisation du système donne le sentiment de se conformer à une norme sociale sans le vouloir. Les attentes sociales poussant à publier plus et plus vite au "risque d'être oublié " génèrent également un inconfort émotionnel. Et même si l'artiste répond à ces attentes en étant "plus visible", de nouveaux risques apparaissent alors. Les plus perturbants pour ces artistes sont la perte de contrôle, la "perdurabilité » de traces dévalorisantes, la dépossession de ses travaux (plagiats), la dissolution de la frontière entre les sphères privée/professionnelle, l'exposition aux critiques des internautes voire la futilité de son image. Ces remarques lorsqu'elles sont négatives et répétitives peuvent même conduire à une situation de mal-être au travail. Les propos de Marie (plasticienne) illustrent ces risques :

«personnellement ça m'avait atteint. (...) Je me suis déconnectée de tout ça, j'ai arrêté (...) la création aussi. Je me suis arrêtée de créer. Parce que c'était des remarques, je me suis dit que peut-être cette opinion était partagée et bon, parce que c'était très précis, il y a des moments où c'était très précis, hein. Je me suis dit peut être que ça plaît pas, peut être que je suis dans la mauvaise direction (...) ça peut en venir à chambouler tes convictions personnelles ».

La dimension "personnelle émotionnelle» et la dimension «interpersonnelle » se rejoignent particulièrement lorsque les artistes questionnent l'authenticité et la sincérité des relations sur les RSN. Ils craignent d'être contactés à des fins utilitaristes plutôt que pour leurs compétences artistiques singulières, ils remettent d'ailleurs en question le terme d'«ami » utilisé sur les RSN pour son incohérence avec ce que représentent réellement les contacts professionnels. Plus à la marge, nous avons également identifié deux autres facteurs « interpersonnels» susceptibles d'entraver « l'acception » des RSN. Tout d'abord, le besoin de recourir parfois à la sous-traitance (professionnelle ou amicale) pour la gestion informatique de la "mise en visibilité » sur les RSN peut constituer une gêne. Enfin, lorsque l'artiste ne ferme pas sa connexion, la manifestation sonore du RSN (un «bip») peut être inopportune si cela interrompt un moment interpersonnel 
spécifique (par exemple, lors d'un moment privilégié de création au cours duquel l'artiste sculpteur et son modèle sont en relation).

\subsubsection{Dimension « métapersonnelle » : des règles prescrites par le système qui hiérarchisent et contraignent la « visibilité »}

Le RSN agit comme un système prescriptif qui contraint et hiérarchise la « visibilité ». Le fonctionnement des RSN induit des règles et des manières de publier qui bien qu'elles ne soient pas explicitement énoncées, ni formellement imposées, vont cependant bien affecter l'activité des artistes. Ces règles sont suggérées, notamment, par le biais de rappels personnalisés qui alarment sur la baisse de fréquentation de la page de l'artiste (ex: statistiques et alertes Facebook). Les utilisateurs se sentent contraints d'accélérer la cadence de publication pour qu'une "page dure, et qu'on soit suivi», selon les propos de Sonia (artiste peintre). Cependant, quand bien même l'artiste utilisateur se plie à ces règles sous-tendues par les RSN, cela ne garantit absolument pas que sa « visibilité » soit effective et durable. Cela est d'autant plus vrai pour les arts alternatifs qui demeurent moins visibles que des arts plus « grand public » (Ben, artiste numérique) ou qui trouvent difficilement une place sur l'espace numérique (par exemple, la musique acousmatique, pour être exprimée pleinement, suppose une spatialisation du son). On constate donc une hiérarchisation de la « visibilité » sur les RSN, ce qui peut freiner leur « acceptation ». Les artistes ont finalement peu de contrôle sur les traces numériques les concernant, ils ne parviennent pas facilement à être et à rester "visibles» sur les RSN. Par ailleurs, lorsqu'ils éprouvent le besoin de devenir intentionnellement «moins visibles", comme, par exemple, quand ils souhaitent effacer un commentaire trop négatif, l'opération échappe là encore à leur plein contrôle. Marie (plasticienne) et Télia (chanteuse jazz) énoncent respectivement : "C'est une fenêtre qu'on ne maîtrise pas du tout (...) ouverte » sur laquelle les messages sont difficiles à effacer; quand toutefois il est possible d'agir, la temporalité fait obstacle : «Dans tous les cas ce sera vu parce que le temps qu'on réagisse (...) ».

\subsubsection{Dimension « transpersonnelle » : au risque d'une activité de création « empêchée "}

$\mathrm{Au}$ sein de la dimension "transpersonnelle ", différents facteurs entravent l'acceptation des RSN. Pour commencer, les artistes estiment qu'un RSN véhicule une image moins professionnelle qu'un site internet. Mais surtout, la pression à publier (et à produire plus, pour toujours publier plus) fait peser différents risques de perte de sens au travail, d'amoindrissement de la qualité des productions et de "leur mise en visibilité", d'affaiblissement de la reconnaissance professionnelle et sociale. Ces risques se traduisent aussi par un engagement non souhaité dans une démarche s'apparentant à de la production marchande de l'art, ce qui leur semble peu « acceptable » au regard du métier. Ensuite, l'analyse met en évidence que les pratiques et contenus sur les RSN sont parfois insatisfaisants qualitativement, soit sur le plan esthétique, soit parce qu'ils ont fait l'objet de copies, de piratage, de retouches... Cela est vécu, par les artistes, comme un nonrespect des règles de métier et entraine donc possiblement vers la dévalorisation sociale du métier d'artiste et/ou des œuvres (qui, déjà, souffrent de peu de reconnaissance). Finalement, la possibilité de construire une identité artistique singulière et distinctive se trouve limitée de même que la possibilité de faire reconnaître le métier d'artiste professionnel. 
Le principal facteur « transpersonnel » entravant « l'acceptation » des RSN par les artistes est lorsque l'usage des RSN empêche le développement de l'activité de création. Dans l'ensemble des entretiens, l'usage des RSN est décrit comme étant très chronophage. Le temps alloué dans leur agenda à la création au sens strict est déjà minime parce que des activités connexes doivent être réalisées (recherche de financement ou de lieu d'exposition, emploi alimentaire, "réseautage »...). Or, si le coût temporel lié à l'utilisation des RSN empiète sur le peu de temps de création restant, cela crée de "l'activité empêchée ». Pour Steven (artiste musicien numérique) : « il faut trouver le juste équilibre. Sinon, 15 à $20 \%$ de temps créatif qui restent, internet peut très vite les bouffer. » Ceci semble d'autant plus fort quand une forme d'addiction au RSN se développe. Par exemple, Christelle (artiste peintre) déclare être « devenue accroc », et Serge-Emmanuel (sculpteur) décrit :

«J'attendais des messages, le petit rond rouge en haut. J'étais dessus et je faisais rien. (...) le matin c'était la première chose que je faisais. ...). Mais, je me suis aperçu que c'était n'importe quoi. C'est tellement superficiel (...) ça me prenait du temps. Ça me prenait moi, ça me prenait de l'énergie. (...) je me soulais avec. »

Ainsi, l'usage des RSN au risque d'une activité de création « empêchée » est peu (ou pas) «acceptable».

\subsection{AXE 4 : Stratégies de régulation}

Le travail de thématisation a fait émerger différents types de stratégies de régulation mises en œuvre par les artistes pour faire face aux difficultés rencontrées lorsqu'ils « (se) mettent en visibilité » sur un RSN. En regard de l'analyse de nos résultats, nous proposons quatre catégories de stratégies principales: de résolution de problème, défensives, de rupture et narratives.

\subsubsection{Stratégies de résolution de problème}

Lorsque les artistes font face à une difficulté liée à l'usage des RSN et qu'ils l'identifient, ils peuvent construire et déployer une "stratégie de résolution de problème ». Ce type de stratégie repose sur quatre actions: 1 ) se fixer des buts précis et raisonnés quant à la manière d'utiliser le RSN, 2) choisir de ne consulter et publier que les informations pertinentes au regard de ces buts, 3) avoir anticipé la survenue possible de difficultés nouvelles, et enfin 4) prévoir les solutions correctives le cas échéant.

51 Prenons l'exemple de Serge-Emmanuel (sculpteur) pour illustrer les deux premières actions. Il déclare :

« quand je suis revenu sur facebook c'est parce que vraiment, j'avais l'intention de l'utiliser. Voilà. Comme un moyen (...). J'avais quand même envie d'utiliser facebook pour mes stages, des conférences que j'organise avec une amie thérapeute (...). Et puis toutes les fois, ça me fait plaisir, je mets une pièce dessus. Mais j'en mets très rarement. Ou alors, je dis que j'en ai mis une sur le blog. Ça fait des visites sur le blog comme ça. »

Cet artiste se donne le but d'utiliser le RSN comme un instrument au service de la " visibilité des stages ", de son blog (qu'il valorise bien plus que son profil facebook parce qu'il cherche à les développer) et éventuellement de certaines œuvres qu'il affectionne. Pour réaliser ses buts, il sélectionne le type de contenus qu'il souhaite diffuser : annonces d'évènements, lien vers le blog et éventuellement une photographie d'une œuvre. S'il a élaboré cette stratégie c'est pour gérer le temps alloué à l'utilisation du RSN afin qu'il ne 
prenne pas le pas sur le temps dédié à des activités plus riches de sens (la création, notamment), et aussi pour se prémunir d'une fusion entre sa communication professionnelle et personnelle.

Pour Christelle (artiste peintre), une des problématiques est de jouer le jeu de l'injonction de publication induite par les RSN pour «rester visible». Elle se fixe donc le but de publier régulièrement du contenu. Cependant, elle anticipe la limite de cette stratégie qui serait, selon elle, de lasser le public ce qui, in fine, desservirait «sa visibilité ». Afin de maintenir l'intérêt des internautes, elle prévoit donc une solution qui consisterait à varier les modalités de sa présence numérique en plus d'augmenter le rythme de ses publications :

«je pourrais aussi zoomer au lieu de faire une photo simple d'un tableau entier, juste pour redonner envie aux gens d'aller voir la page ».

\subsubsection{Stratégies défensives}

Les discours donnent à entendre des processus qui s'apparentent aux «stratégies de défenses psychiques", étudiées notamment par Dejours (1995). Procédé mental le plus souvent inconscient, le processus de défense psychique est défini comme « une révolte du moi contre des affects pénibles dans le but d'éviter un déplaisir et d'assurer son intégrité et sa sécurité. » (Alderson, 2004, p. 251). Nos analyses permettent d'identifier trois types de stratégies défensives. Premièrement, les comportements numériques aversifs sont soit rationalisés, soit justifiés en se référant à la logique et au bon sens. Pour Liam (chanteurcompositeur):

« je ne dois pas avoir beaucoup de talent, je m'en fous. J'ai eu des messages "ouais bof moyen", "les textes sont sympas, la musique dégueulasse", des fois l'inverse, normal, on ne peut pas plaire à tout le monde. »

Deuxièmement, la reconnaissance du réel consiste à admettre que certaines difficultés liées à l'usage des RSN se rapportent en fait de façon inhérente et inévitable à la situation globale de leur développement artistique actuel. Puisque ces difficultés vécues sur l'espace numérique s'inscrivent immanquablement dans la situation réelle, alors l'artiste gagne à les reconnaître pour les relativiser et les accepter plutôt que les subir. Considérons le cas d'un artiste qui craint d'être oublié parce qu'il n'a pas de contenu à publier sur le RSN, reconnaître le fait que cette absence de contenu est en fait cohérente avec son activité artistique réelle peut atténuer la difficulté émotionnelle. Au moment de l'entretien avec Christelle (artiste peintre), «il ne se passe plus rien» sur son espace Facebook, cela l'angoisse, elle cherche comment remédier à cette baisse d'audience ( $«$ il faudrait poster quelque chose toutes les semaines, tous les jours »). Et finalement, elle reconnaît le réel : cette tension est inévitablement liée au fait qu'elle participe à peu d'événements à ce moment ("c'est vrai qu'en ce moment j'ai pas d'actualité, pas d'annonce d'expo pour l'instant, donc c'est moins intéressant. »), mais aussi à un fonctionnement habituel («je suis dans un état presque végétatif. Niveau création. C'est un phénomène récurrent. Après expo, je fais des micro-dépressions. Je me mets en mode repos, vacances, en fait, non, parce que ça n'arrête pas de cogiter, mais sur le terrain, je fais plus rien »).

L'auto-attribution des causes d'une difficulté est la troisième défense mise en évidence dans l'analyse. Elle correspond au fait de s'attribuer la responsabilité des difficultés liées à l'usage des RSN. Ce mécanisme entrave inévitablement leur « acceptation ». Par exemple, les entretiens avec Serge Emmanuel (sculpteur) et Christelle (peintre) illustrent comment ces artistes s'attribuent la cause d'une utilisation excessive des RSN au détriment de leur 
activité créative. Christelle énonce "je suis devenue un peu accroc » et «la contrainte, c'est moi qui me la mets d'y aller tous les jours et de perdre du temps. (...) de passer trop de temps.» Cette situation génère un inconfort émotionnel, car cela lui renvoie une image d'ellemême négative et dissonante par rapport à ses représentations initiales :

« je me disais tout le temps "ces gens-là, ils sont toujours avec leur téléphone, leur truc, mais ils font quoi, ils travaillent pas, ils font rien?". Et là je fais la même chose."

Des stratégies collectives sont également possibles. Par exemple, écrire au nom d'un collectif de pairs plutôt qu'en son nom propre d'artiste peut être une manière de se protéger, soi et son intimité.

\subsubsection{Stratégies de rupture}

Les artistes opèrent différentes formes de rupture avec les RSN qui peuvent se traduire par une prise de distance entre l'artiste et le réseau, mais aussi par des procédés de (re)matérialisation.

Nous constatons que cette distanciation peut s'opérer de manière radicale (l'artiste interrompt son usage des RSN parfois pendant plusieurs années) ou partielle (l'artiste est utilisateur, mais développe des pratiques d'anonymisation, l'absence de photographie ou de tout autre élément d'identification, créant de ce fait une rupture entre son identité civile et son identité numérique). Cette stratégie de distanciation peut aussi se traduire par moins d'implication émotionnelle : l'artiste utilisateur évite d'écrire ou de publier des contenus à forte valeur affective. Cela faciliterait le détachement et limiterait la confrontation à une charge émotionnelle.

Le recours au matériel est la seconde modalité des stratégies de rupture de «(re)matérialisation». Afin de rompre avec les tensions générées dans le «monde numérique », les artistes peuvent recourir au « monde réel, matériel ». Par cette stratégie, aucun contenu publié sur le RSN n'appartient strictement au monde numérique. Par exemple, pour faire face au risque de dégradation ou de plagiat d'une œuvre, une partie des artistes opte pour publier uniquement l'image d'œuvres ayant déjà une "visibilité " dans le monde réel (ce peut être une toile déjà exposée). Nous avons aussi repéré une stratégie qui est d'associer systématiquement les messages numériques à des rendez-vous " physiques » (une assemblée générale, un concert, par exemple).

Ces stratégies de rupture avec l'espace numérique pour réintroduire le primat des objets et interactions physiques semblent donc soutenir a minima les artistes dans la protection des œuvres et du métier ainsi que dans le développement d'une communication utile qui limite le risque de se disperser et de perdre du temps avec l'utilisation du RSN.

\subsubsection{Stratégies narratives}

Les artistes peuvent avoir recours à des stratégies de narration, qu'ils adressent aussi bien à eux-mêmes qu'à autrui (au public, notamment).

Dans le cadre de narrations adressées à eux-mêmes, les artistes se représentent le public. Ils questionnent puis construisent l'histoire de leur audience. Comment ces internautes sont-ils parvenus jusqu'à leur page? Quelles sont leurs motivations ? Sont-ils touchés par les œuvres visibles sur le RSN? Ainsi, tous les internautes ne prennent pas la même valeur aux yeux de l'artiste et, de ce fait, leurs commentaires ne les touchent pas de la même manière. Cette stratégie préserve l'artiste sur le plan émotionnel, mais lui permet 
aussi de faire un usage différencié et spécialisé des différents espaces numériques sur lesquels il se trouve visible. Selon les chemins présumés de la démarche des internautes, il en résulte, par exemple, un plus fort investissement des artistes envers les formes et contenus de visibilité au sein de groupes restreints, composés d'internautes qui ont certainement eu une démarche de recherche active qui témoigne d'un profond intérêt pour l'artiste.

Concernant la narration adressée à autrui, il s'agit en quelque sorte de faire entendre la voix du personnage artistique créé ou leur voix de professionnel. Les récits et contenus édités sont construits pour être cohérents avec ce personnage ou professionnel. Un effort stylistique et esthétique est produit (poésie ou philosophie, par exemple) dans le but d'enrichir ce personnage, de le rendre imaginable et facilement identifiable par le public. La teneur du message est parfois enjolivée. Des parts peu valorisantes du réel de l'activité de l'artiste peuvent être occultées (e.g. on n'écrit pas qu'il y a eu peu de participants à un événement) ou à l'inverse il est possible d'exacerber les qualités du personnage (e.g. prôner des principes de vie sans les appliquer réellement). Cette stratégie de narration adressée à autrui se place donc en réponse aux besoins de contrôler «sa mise en visibilité ", de publier des traces numériques valorisantes pour sa propre activité et pour la profession au sens général, mais aussi de préserver une frontière entre "soi-artiste professionnel visible » et « soi-intime invisible».

Cette narration de soi, adressée à autrui, est particulièrement soutenue par un mouvement réflexif. La réflexivité correspond à une prise de conscience de soi. Par ce biais, les artistes parviennent à distinguer leur soi-intime de leur soi-artiste, ils régulent ainsi les relations entre ces deux entités identitaires complémentaires, mais différentes. Avoir conscience de son soi-intime, de ses limites personnelles ou encore de ses émotions est une protection face aux difficultés identifiées dans la dimension personnelle, notamment le risque de surexposition de soi. De plus, avoir conscience de son soi-artiste soutient l'élaboration des discours et autres formes de visibilité professionnelle sur les RSN. En effet, il devient plus facile d'identifier et de valoriser les éléments singuliers de l'artiste. Les traces laissées sur l'espace numérique se révèlent être comme un miroir dans lequel l'artiste peut se regarder être artiste avec un certain recul. Cette forme d'autoconfrontation réflexive permet alors de développer son identité professionnelle sur et par le réseau numérique.

\section{Synthèse et perspectives}

Partant du constat que « la visibilité » et « l'invisibilité » représentent à la fois des enjeux et des risques pour l'artiste, cette recherche s'est portée sur l'identification des facteurs favorables «d'acceptation» des RSN comme instruments médiateurs de l'activité «de mise en visibilité ». Les freins et leviers « d'acceptation » des RSN ont été ordonnés selon les composantes "personnelle, interpersonnelle, métapersonnelle et transpersonnelle " du modèle de « l'acceptation située » des technologies (Bobillier Chaumon 2013, 2016).

L'analyse confirme l'importance de « la visibilité » pour les artistes. La "mise en visibilité de soi» et de ses œuvres peut être un moyen de construction identitaire, de reconnaissance sociale et de promotion favorable à la carrière de l'artiste. Les artistes interrogés, utilisateurs ou non, novices ou experts, s'accordent tous pour reconnaître la nécessité professionnelle d'utiliser les RSN. Le principal moteur de «l'acceptation » se trouve dans la composante « interpersonnelle». Cela est cohérent avec le fait que les 
«fonctions constituantes » (Rabardel, 1995) prévues initialement par les concepteurs des RSN, visent d'abord la communication. Sur les RSN, les artistes « résautent » et cherchent à valoriser leur travail dans un rapport plus direct, bien que médiatisé, avec l'ensemble des acteurs culturels. Ce mouvement, parfois qualifié de "désintermédiation sauvage " (Bacache, et al., 2009, p.13), correspond à la nécessité pour l'artiste de développer toujours plus d'interactions avec le public (Le Coq, 2004), car « la capacité de créer va de pair avec la capacité de créer le public (...). » (Kridis, 2010, p. 139).

La prégnance de la dimension "personnelle » ressort en quatre endroits de l'analyse : au travers des leviers "d'acceptation", des facteurs de rejet des RSN, des motifs pour lesquels développer des stratégies de régulation et enfin comme élément fondamental de la définition du métier d'artiste, métier "de l'être et pas seulement du faire " selon Florie (sculptrice). La saillance de cette dimension est cohérente avec la littérature qui a indiqué que la composante personnelle du métier est la plus investie par les artistes (engagement, effort de singularisation, vocation voire passion), mais aussi dans l'imaginaire social du public.

Les principaux freins à «l'acceptation» des RSN renvoient respectivement aux dimensions «personnelle, métapersonnelle et transpersonnelle» du modèle "d'acceptation située » des technologies (Bobillier Chaumon, 2013). Un sentiment d'intensification du travail côtoie une tension émotionnelle qui se joue dans une situation paradoxale: la peur d'être oublié pousse à publier davantage, mais publier davantage expose à de nouveaux risques (être plagié, critiqué, mêler privé et professionnel ou perdre en authenticité dans les relations établies avec les " amis virtuels»). Des règles induites par le RSN contraignent la « visibilité » et conduisent là encore l'artiste à publier toujours plus, mais sans lui laisser pour autant le plein contrôle des traces déposées sur l'espace numérique. Cette situation génère un conflit de valeurs : produire des œuvres « à la chaîne " reviendrait à trahir un accord générique (au sens de "genre professionnel » selon Clot, 2008) qui veut, notamment, que l'on préserve la liberté de l'artiste et la rareté des œuvres. En outre, ces pratiques mettent parfois à mal l'identité et le «style» professionnels, le sens du travail artistique et enfin, elles «empêchent» (Clot, 2008) l'activité de création.

68 L'activité de création empêchée par l'usage des RSN est un des freins à leur « acceptation » qui motive le plus de recourir à des stratégies de régulation. Ces stratégies consistent en des actions de résolution de problème, de protection (stratégies défensives et stratégies de rupture) ou de développement par la narration et la réflexivité. Notons tout de même que l'effort de régulation ne dépend pas uniquement de l'artiste, mais procède d'une "coproduction où se rencontrent les stratégies des plateformes et les tactiques des utilisateurs. » (Cardon, 2008, p. 97). Cette catégorisation ne revendique pas l'exhaustivité, elle invite, au contraire, à poursuivre la recherche en ayant recours à d'autres méthodes complémentaires. Par exemple, des démarches d'analyse réflexive de l'activité et d'élaboration collective, de type objet technique (Simondon, 1959) ou des dispositifs d'autoconfrontations croisées (Clot, Faïta, Fernandez, \& Scheller, 2000) permettraient une mise en débat des différentes manières de gérer les tensions liées à l'usage des RSN entre professionnels partageant des référentiels de métier.

69 Au-delà d'une compréhension de « l'acceptation » des RSN au travers de ses déterminants et stratégies, l'étude réalisée peut aussi participer au développement professionnel des artistes. Nous proposons qu'un des leviers de développement intéressant dans le cadre de ce métier se situe sur le plan de la réflexivité et de l'esthétique de la narration numérique. 
Les stratégies narratives, en même temps qu'elles soutiennent «l'acceptation et l'appropriation » des RSN comme instruments « de mise en visibilité », créent aussi une occasion de développement par le recours à la créativité. En effet, la narration de soiartiste engage d'abord un travail réflexif pour élaborer, conscientiser et expliciter son identité artistique singulière. L'artiste développe alors des compétences nécessaires à la présentation de soi et enrichit son image artistique. En ce sens, les constructions narratives des artistes sur les RSN peuvent constituer des propos esthétiques et artistiques à part entière, elles représentent donc un intérêt certain pour le sujet et le métier.

Comme le montre Boucharon (2008, p. 143), les supports numériques interactifs sont propices à un travail esthétique dont la visée «couvre aussi bien le poétique dans le domaine du langage que la figure dans le domaine de la plastique » et « est indissociable d'une esthétique de la matérialité (du texte, de l'interface et du support)». En d'autres termes, les RSN offrent de multiples possibilités textuelles (ex : dialogues, coproduction de texte avec le public...), spatiales (ex : usage de la 3D, liens hypertextes, environnements immersifs...), temporelles (instantanéité, traces mémorisées vs éphémères...) ou encore matérielles (ex : images, vidéos...) qui peuvent être combinées dans une visée esthétique singulière. Une perspective d'intervention favorable à « la mise en visibilité » des artistes sur les RSN consisterait en l'accompagnement à l'usage esthétique de la narration numérique. Plus généralement, une sensibilisation à l'identité et «la visibilité numérique » pourrait être offerte aux étudiants des écoles d'art, mais également dans le cadre de rencontres et de débats entre scientifiques et artistes. La recherche menée a d'ailleurs été saisie par un duo d'artistes pour organiser une exposition de leurs œuvres, une discussion avec les chercheurs ainsi qu'une soirée-débat philosophique. Ainsi, si l'objectif initial poursuivait une visée de recherche, des effets de transformations ont ainsi pu être observés.

\section{BIBLIOGRAPHIE}

Alderson, M. (2004) La psychodynamique du travail : objet, considérations épistémologiques, concepts et prémisses théoriques. Santé mentale au Québec, 29(1), 243-260.

Bacache, M., Bourreau, M., Gensollen, M., \& Moreau, F. (2009). Musiciens dans la révolution numérique : inquiétude et enthousiasme. Paris : Centre d'Information et de Ressources pour les Musiques Actuelles.

Bain, A. (2005). Constructing an artistic identity. Work, Employment and Society, 19(1), 25-46. DOI: 10:1177/0950017005051280.

Bobillier Chaumon, M.E. (2013). Conditions d'usage et facteurs d'acceptation des technologies dans l'activité : questions et perspectives pour la psychologie du travail. Mémoire d'habilitation à diriger des recherches. Grenoble : Université Pierre Mendès France.

Bobillier Chaumon, M.E. (2016). Acceptation située des TIC dans et par l'activité : Premiers étayages pour une clinique de l'usage. Psychologie du Travail et des Organisations, 23, 4-21. 
Bobillier Chaumon, M.E., Carvallo, S., Tarpin-Bernard, F., \& Vacherand-Revel, J. (2005). Standardiser ou adapter les interactions personnes-machines? Revue d'Interaction Homme Machine / Journal of Man-Machine Interaction, 2(2), 91-129.

Bobillier Chaumon, M.E., \& Dubois, M. (2009). Synthèse introductive : l'acceptabilité des technologies : bilans et nouvelles perspectives. Travail Humain, 72(4), 305-310.

Bonnardel, N. (2009). Activités de conception et créativité : de l'analyse des facteurs cognitifs aux activités de conception créatives. Le Travail Humain, 72, 5-22.

Boucharon, S. (2008) Le récit littéraire interactif : Une esthétique de la matérialité. In M. Maza \& A. Saemmer (Eds.), E-formes : écritures visuelles sur support numérique (pp. 135-144). Saint-Étienne : Publications de l'Université de Saint-Étienne.

Bourdieu, P. (1979). La distinction, critique sociale du jugement. Paris : Éditions de Minuit.

Brodin, O., \& Magnier, L. (2012). Le développement d'un index d'exposition de soi dans les médias sociaux : phase exploratoire d'identification des indicateurs constitutifs. Management \& Avenir, 58, 144-168. DOI : 10.3917/mav.058.0144.

Buscatto, M. (2004). De la vocation artistique au travail musical : tensions, compromis et ambivalences chez les musiciens de jazz. Sociologie de l'Art, 3 OPuS 5, 35-56. DOI : 10.3917/ soart.005.0035.

Buscatto, M. (2006). Ethnographies du travail artistique : apports et usages épistémologiques. Sociologie de l'Art, 2 OPuS 9 \& 10, 87-105. DOI : 10.3917/soart.009.0087.

Cardon, D. (2008). Le design de la visibilité : un essai de typologie du web 2.0. http:// www.internetactu.net/2008/02/01/le-design-de-la-visibilite-un-essai-de-typologie-du-web-20/. Clot, Y. (1999). La fonction psychologique du travail. Paris : PUF, collection Le Travail Humain. Clot, Y. (2008). Travail et pouvoir d'agir. Paris : PUF.

Clot, Y., Faïta, D., Fernandez, G., \& Scheller, L. (2000). Entretiens en autoconfrontation croisée : une méthode en clinique de l'activité. Perspectives interdisciplinaires sur le travail et la santé, 2(1), http://pistes.revues.org/3833.

Croissant, V., \& Touboul, A. (2011). Le multimédia ou le langage des sociabilités numériques : l'exemple de la communication des artistes sur les réseaux sociaux numériques. http://w3.ugrenoble3.fr/les_enjeux/2011-dossier.

Csikszentmihalyi, M. (1996). La créativité, psychologie de la découverte et de l'invention. Paris : Robert Laffont.

Dejours, C. (1995). Le facteur humain. Paris : PUF.

Donnat, O. (2009). Les passions culturelles, entre engagement total et jardin secret. Réseaux, 153, 79-79. DOI : 10.3917/res.153.0079.

Fallery, B., \& Rodhain, F. (2007). Quatre approches pour l'analyse de données textuelles : lexicale, linguistique, cognitive, thématique. XVİ̀me Conférence de l'Association Internationale de 56 Management Stratégique AIMS, Montréal. En ligne : http://hal.archives-ouvertes.fr/hal-00821448. Flichy, P. (2010). Le sacre de l'amateur : sociologie des passions ordinaires à l'ère du numérique. Paris : Seuil.

Fourmentraux, J.P. (2008). La création au risque d'Internet, Mouchette (1996-2006) : œuvre ou artiste ? Ethnologie française, 38, 59-68. DOI : 10.3917/ethn.081.0059. 
Fourmentraux, J.P. (2010). Art et internet : les nouvelles figures de la création. Paris : CNRS Éditions.

Goffman, E. (1973). La mise en scène de la vie quotidienne. (Tome 1 : Présentation de soi et tome 2 : Relations en public). (Éds org., 1959). Paris : Éditions de Minuit.

Heinich, N. (1995). Façons d' » être » écrivain. L'identité professionnelle en régime de singularité. Revue française de sociologie. 36(3), 499-524.

Kridis, N. (2010). Psychologie de l'artiste créateur. Paris : L'Harmattan.

Le Coq, S. (2004). Le travail artistique : effritement du modèle de l'artiste créateur ? Sociologie de l'Art, 3 OPuS 5, 111-131. DOI : 10.3917/soart.005.0111.

Norman, D.A. (1993). Les artefacts cognitifs. (Traduction française : Francesco Cara). Raisons pratiques : les objets dans l'action, 4,15-34.

Paillé, P., \& Mucchielli, A. (2012). L'analyse qualitative en sciences humaines et sociales. Paris : Armand Colin.

Quitaud, G. (1993). Le voyage vers l'œuvre : les liens multiples unissant l'Homme au processus créatif. Toulouse : Erès.

Rabardel, P. (1995). Les Hommes et les technologies, une approche cognitive des instruments contemporains. Paris : Armand Colin.

Rouquette, M.L. (2007). La Créativité. Paris : PUF.

Simondon, G. (1959) Du mode d'existence des objets techniques. Paris : Aubier.

Stenger, T., \& Coutant, A. (2010). Les réseaux sociaux numériques : des discours de promotion à la définition d'un objet et d'une méthodologie de recherche. Journal of language and communication studies, 44, 209-224.

Therriault, G., \& Harvey, L. (2011). Postures épistémologiques que développent de futurs enseignants de sciences et de sciences humaines lors des cours de formation disciplinaire et pratique : l'apport d'une recherche mixte. Recherches qualitatives, 30(2), 71-95.

Vermersch, P. (1994). L'entretien d'explicitation. Issy-Les-Moulineaux : ESF Édition.

Zammar, N. (2012). Réseaux sociaux numériques : essai de catégorisation et cartographie des controverses. Thèse de doctorat en sciences de l'information et de la communication, Université Rennes 2.

\section{NOTES}

1. Ce travail s'intègre au projet «L'Avis des Autres » coordonné par l'Université Lyon 2. Il s'agit d'une recherche pluridisciplinaire sur la thématique des prescriptions culturelles et numériques. La contribution des chercheurs en psychologie du travail a consisté en une analyse des apports des RSN dans la mise en œuvre et le développement de l'activité artistique et culturelle. Cette recherche ne porte pas intrinsèquement de visée de transformation.

2. Les déterminants environnementaux et leurs impacts sur la création sont étudiés, par exemple, dans les travaux de Csikszentmihalyi (1996).

3. Notre traduction de " a distinctive and marketable identity ».

4. Il est à noter que la catégorisation des «formats de visibilité » sur les RSN proposée par Cardon (2008) n'a pas été développée spécifiquement en référence à une population d'artistes, le format « phare » peut donc être appliqué pour différents contextes d'usage et de populations. 
5. Notons que cette recherche auprès d'artistes a placé les chercheurs en présence d'une activité professionnelle difficile à appréhender. Pour une meilleure compréhension du "réel» de l'activité artistique, des gestes, du sens ou encore des mots de cette profession, une expérimentation de la tâche de création, une auto-confrontation simple et une autoconfrontation collective ont été menées auprès de trois sculpteurs et d'un photographe. Ces travaux ne sont pas présentés dans le présent article.

\section{RÉSUMÉS}

Les Réseaux Sociaux Numériques représentent a priori des instruments médiateurs de l'activité, essentielle, de «mise en visibilité » des artistes. Mais l'usage de ces dispositifs peut aussi être une contrainte ou un empêchement. Aussi, cette étude poursuit le but d'identifier les facteurs favorables et défavorables à l'acceptation des RSN par les artistes. Le cadre théorique de "l'acceptation située " des technologies selon Bobillier Chaumon (2013) est mobilisé pour soutenir une démarche de recherche qualitative auprès de 15 artistes numériques et traditionnels. L'analyse thématique de 12 entretiens semi-directifs et 4 entretiens à visée d'explicitation révèle que le processus «d'acceptation» des technologies s'inscrit dans un rapport aux technologies déjà existant qui dépend des expériences vécues avec des dispositifs technologiques et de la façon dont la technologie s'ancre dans la démarche artistique. Ce processus est modulé par des leviers principalement rattachés aux dimensions «personnelle et interpersonnelle » du modèle « d'acceptation située », et des freins se rapportent principalement aux dimensions "personnelle, transpersonnelle et métapersonnelle». Nous avons identifié quatre types de stratégies de régulation déployées pour faire face aux freins. Les stratégies qualifiées de «narratives » représenteraient un intérêt spécifique pour l'acceptation du RSN et pour la profession d'artiste en sollicitant créativité et esthétique.

Improving the visibility of art(ists) is an essential activity which could be mediated by Social Networks Sites (SNS). However, SNS represent both a resource and a constraint for artists. We therefore argue that it is not a straightforward process for artists to accept these technologies. The aim of this study is to identify factors facilitating or impeding the acceptance of SNS by artists. The theoretical framework supporting this research is the situated technology acceptance model (Bobillier Chaumon, 2013). This model proposes to analyse the acceptance of technology through personal, interpersonal, metapersonal and transpersonal dimensions. We conducted a qualitative research with both traditional and digital artists. The method was based on 12 semi-structured interviews and 4 interviews for explanatory purposes. The results highlight the influence of attitudes to new technologies and the consistency between technologies and artistic direction on the SNS acceptance process. Factors facilitating SNS acceptance mainly embody interpersonal and personal dimensions. Transpersonal, metapersonal and personal dimensions comprise negative factors which can lead to a rejection of SNS. We identify 4 types of regulation strategy to cope with negative factors. Among these strategies, we discuss the utility of narrative strategies for the development of both SNS acceptance and artist development. 


\section{INDEX}

Mots-clés : (in)visibilité artistique, Réseaux Sociaux Numériques (RSN), acceptation des technologies, stratégies de régulation, narration numérique

Keywords : art (in)visibility, social network sites (SNS), technology situated acceptance, regulation strategy, digital storytelling

\section{AUTEURS}

\section{AMANDINE PORCHER}

Université Lyon 2 - Institut de Psychologie - Laboratoire GRePS porcher.amandine@gmail.com

\section{JACQUELINE VACHERAND-REVEL}

École Centrale de Lyon - Laboratoire GRePS

jacqueline.vacherand-revel@ec-lyon.fr

\section{MARC-ÉRIC BOBILLIER CHAUMON}

Université Lyon 2 - Institut de Psychologie - Laboratoire GRePS marc-eric.bobillier-chaumon@univ-lyon2.fr

\section{MANON MOKTARI}

Université Lyon 2 - Laboratoire GRePS

manon.moktari@univ-lyon2.fr

\section{BRUNO CUVILLIER}

Université Lyon 2 - Institut de Psychologie - Laboratoire GRePS bruno.cuvillier@univ-lyon2.fr 